\title{
Tracheal Stenosis After Endotracheal Intubation and Percutaneous Dilatational Tracheostomy
}

\author{
Camelia Herdini, ${ }^{1}$ Agus Surono, ${ }^{1}$ Supomo Supomo, ${ }^{2}$ Jessica Fedriana ${ }^{1}$
}

\section{Introduction}

\section{$\underline{\text { ABSTRACT }}$}

Tracheal stenosis is an abnormal narrowing of the tracheal lumen which affects adequate airflow and caused by an inflammatory complication such as endotracheal intubation and percutaneous dilatational tracheostomy (PDT). Incidence of tracheal stenosis following endotracheal intubation and PDT was $8-44 \%$.

Case Report

A 24 year old female presented with dyspnea and hoarseness after traffic accident. She was intubated for 2 weeks then followed by PDT for 3 weeks. The laryngoscopy examination after PDT extubation showed tracheal stenosis at the second-third tracheal ring with left vocal fold granuloma. Cervical computed tomography demonstrated a mass at vocal cord and narrowing of tracheal caliber at the first thoracic vertebra disk, above the stoma of PDT.The granuloma was excised and tracheal stenosis was removed by tracheal resectionand end-to-end anastomosis.

Discussion

Tracheal stenosis is one of important sequelae after endotracheal intubationand PDT. Tracheal resection and primary anastomosis may be considered as an option for surgical management of tracheal stenosis.

Kevwords

Tracheal Stenosis

$\mathrm{T}$

racheal stenosis is an abnormal narrowing of the tracheal lumen which can compromise airflow. It is caused by prolonged intubation or tracheostomy, and is complex and difficult to manage. ${ }^{1}$ Oral or nasal endotracheal tubes or tracheostomy tubes are commonly used to render mechanical ventilatory support in respiratory failure. ${ }^{2}$ Despite technological improvements and skillful patient care in intensive care units, laryngotracheal stenosis continues to be common after intubation and tracheostomy. ${ }^{2,3}$ In some cases, tracheal stenosis may occur below the cuff of the tube and above the stoma. ${ }^{3}$

Incidence of tracheal stenosis following endotracheal intubation and percutaneous dilatational tracheostomy (PDT) is difficult to quantify. It has been reported to be $8-44 \%$ by various authors, whereas incidence of symptomatic tracheal stenosis with more than $50 \%$ tracheal narrowing was $2-6 \% .^{4-6}$ The management involves a multidisciplinary approach with multiple complex procedures. ${ }^{1}$

\section{Case Report}

A 24 year old female presented to Dr. Sardjito Hospital in Yogyakarta, Indonesia for evaluation of dyspnea and hoarseness. The patient was treated in intensive care unit following a road traffic accident 3 months back. She was on endotracheal intubation for 2 weeks. Then she underwent a percutaneous dilatational tracheostomy (PDT) and was on tracheostomy for 3 weeks. Two weeks after decannulation, she experienced dyspnoea on exertion and a persistent change in her voice. The initial direct flexible laryngoscopy examination revealed a solitary, round smooth, pedunculated mass in left posterior larynx, which was a suspected vocal fold

1 - Department of ENT, Universitas Gadjah Mada

2 - Department of Surgery, Universitas Gadjah Mada

Corresponding author:

Dr Camelia Herdini

email: camelia_herdini@yahoo.com 

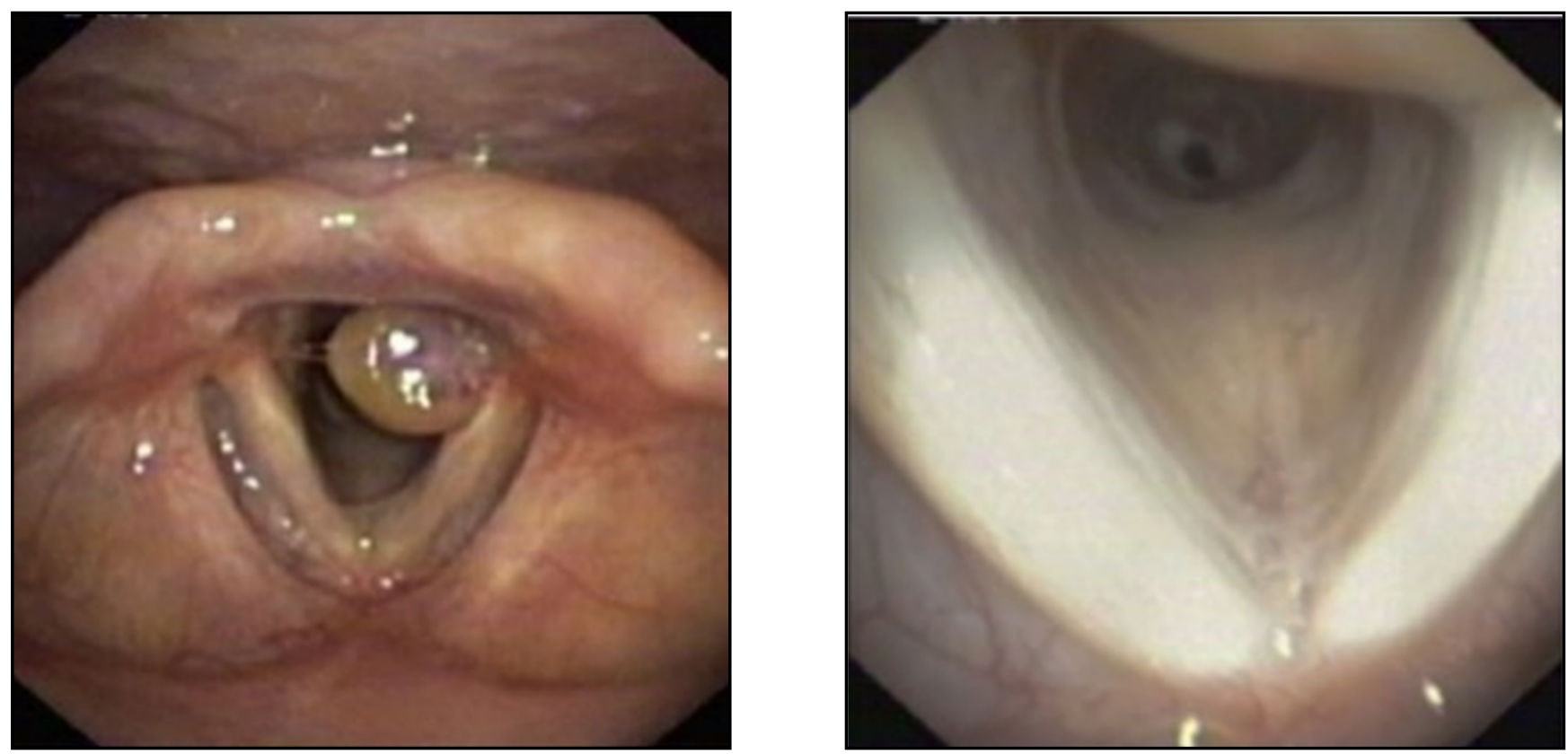

Fig. 1 Initial examination of flexible laryngoscopy. (A) Vocal fold granuloma and (B) tracheal stenosis.

granuloma. Below that mass, there was tracheal stenosis as high as the second-third tracheal ring (Fig. 1). The movement of the vocal folds were relatively normal. There was a surgical scar in the neck and no palpable cervical lymphadenopathy. The patient did not have respiratory distress.

Multi-slice computed tomography (MSCT) of neck demonstrated a narrowing of tracheal calibre as high as the first thoracic vertebra disk with diameter $0.77 \mathrm{~cm}$. Diameter of the proximal normal segment was $1.37 \mathrm{~cm}$ (the stenosis was $56 \%$ of the tracheal diameter). There was a stoma of PDT on anterior tracheal wall as high as second thoracic vertebra corpus (Fig. 2).

Clinical history, physical examination, and imaging

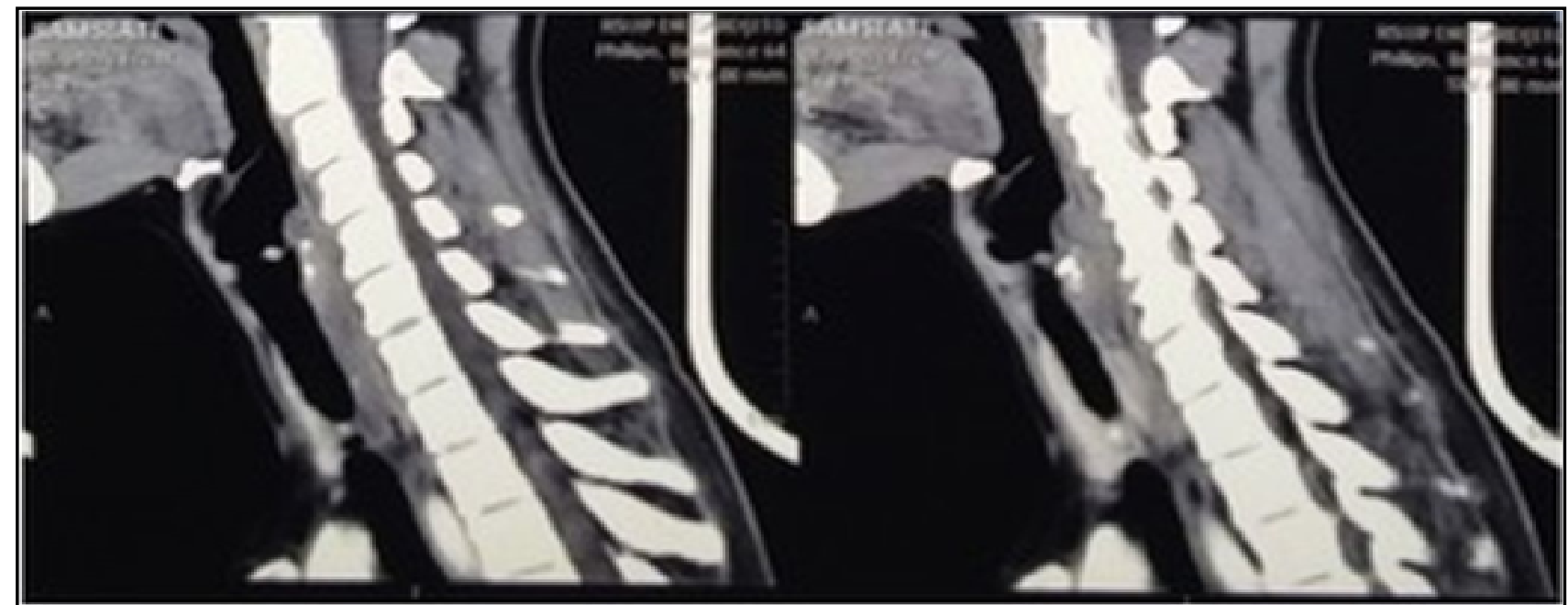

Fig. 2 Multi-slice computed tomography of the cervical. The tracheal stenosis was shown as high as the first thoracic vertebra corpus. 
refer to the diagnosis of tracheal stenosis. The management was resection of the trachea followed by end-to-end anastomosis under general anesthesia. The stenosed second and third tracheal rings were removed. Post operatively, she was on endotracheal intubation for 10 days and was given antibiotics, analgesics, steroids, and proton pump inhibitors. After extubation, she was completely relieved from dyspnea and hoarseness. Post operative flexible laryngo-tracheoscopy revelead shrunken vocal fold granuloma and patent tracheal airway with normal vocal fold movements. 3 months post operatively, the patient remained symptom-free (Fig. 3).

\section{Discussion}

Tracheal stenosis is an abnormal narrowing of the tracheal lumen. It begins with trauma and ulceration of tracheal mucosa with superimposed local infection, leading to perichondritis of tracheal cartilage followed by increased fibroblastic activity leading to circumferential scarring and airway obstruction. ${ }^{1}$ Another postulated causative factor for stenosis is loss or reduction of regional blood flow due to the pressure exerted by the

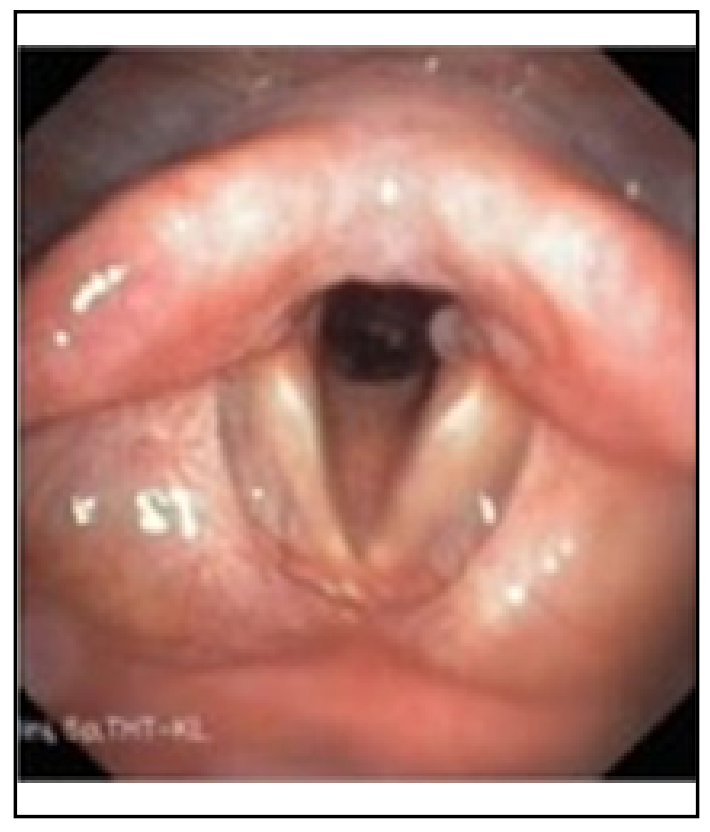

Fig. 3 Examination of direct flexible laryngoscopy after three months of surgery. cuff on the tracheal wall. ${ }^{7}$ The infectious process also leads to formation of granulation tissue that contributes to scarring. Long-term intubation and tracheostomy account for the majority of benign but potentially hazardous tracheal stenosis. ${ }^{4}$

Tracheostomy was originally used strictly for the emergency management of upper-airway obstruction and was therefore associated with a high incidence of serious complications. More recently, it has become an elective procedure intended to improve clearance of secretions and prevent the complications associated with prolonged endotracheal intubation in ventilator dependant or critically ill patients. ${ }^{4}$ Complications of open tracheostomy are more than those of percutaneous procedures, but percutaneous tracheostomy procedures are also associated with some dreaded complications. To prevent cartilage damage, particularly during percutaneous tracheostomy, excessive force must not be applied to the trachea, and the placement of the stoma must be guided by bronchoscopy. Some centres prefer open tracheostomy. ${ }^{8}$

Post intubation stenosis is generally longer and uniform compared to post tracheostomy stenosis which is generally an extension of granulation tissue from the injured anterior wall of the trachea. ${ }^{1}$ The common sites of tracheal stenosis post tracheostomy are the stoma, the supra stomal area, and the cuff site. ${ }^{9}$ In this patient, tracheal stenosis occurred at the supra stomal area at the second and the third tracheal ring. The tracheal stenosis was $56 \%$ of the tracheal diameter. ${ }^{10}$

Although stenosis generally develops within two to three months of decannulation, it can occur as early as two to six weeks. The symptoms of a functionally significant stenosis include dyspnoea on exertion, cough, or inability to clear secretions. Symptoms of stenosis at rest are unusual until at least $75 \%$ of the diameter of the trachea has been obstructed. Critical narrowing with stridor does not occur until the diameter of the tracheal lumen is less than five to six $\mathrm{mm} .{ }^{4}$ This patient experienced dyspnoea on exertion and a persistent change of voice without stridor two weeks after decannulation from PDT.

There are two basic modalities available for treatment of tracheal stenosis, namely endoscopic and external 
(open) surgery. The endoscopic approach includes traditional dilation, laryngeal microsurgery, laserassisted excision, and endoscopic stent placement. ${ }^{11}$ Endoscopic dilation should be reserved for patients with significant comorbidities or as a temporary measure in non-equipped centres. ${ }^{7}$ External surgical reconstruction includes tracheal resection and anastomosis, laryngotracheoplasty, pericardial patch tracheoplasty, slide tracheoplasty, homograft tracheal transplantation, and autologous reconstructed trachea. ${ }^{12}$ Open surgery is recommended for patients with complex stenosis or restenosis after multiple procedures and those in conjunction with associated conditions like tracheoesophageal fistula closure. Contraindications are long segment involvement (more than 50\%), multiple focal involvement, and poor surgical condition. ${ }^{1}$

Resection of the stenosed segment of trachea followed by end-to-end anastomosis has become the standard of care in many centres and has given the most consistent results. It is indicated for tracheal stenosis involving less than two-thirds of tracheal length. ${ }^{12,13}$ Post-operatively broad spectrum antibiotics are usually required to prevent local infection and wound breakdown. Antireflux therapy is also needed to protect the airway. In addition, corticosteroids are recommended in the first 48-72 hours, but they should not be used longer since they may impair healing. Maintaining neck flexion is necessary to prevent suture line disruption. Patient is followed up endoscopically four to six weeks post operatively to check suture lines. ${ }^{12}$ This patient was given antibiotics, analgesics, steroids, and proton pump inhibitors.

According to the literature, the success rate ranges from 71 to $97 \%,{ }^{8}$ with 1.8 to $5 \%$ mortality rate and 5 to $15 \%$ failure rate. ${ }^{14}$ Staged reconstructions were needed in $26 \%$ of the patients. Older patients aged more than 60 years and patients with a higher grade of stenosis showed a significantly lower success rate. Staged operations were more frequently needed in cases with total stenosis and with combined stenosis of the trachea and the subglottis. ${ }^{14}$ Complications of open surgery include vocal cord palsy, restenosis, suture granuloma formation, and infection. ${ }^{1}$ This patient was relieved from dyspnea and hoarseness, without any complication. In conclusion, tracheal stenosis is an important sequel of endotracheal intubation and percutaneous tracheostomy procedures. Tracheal resection and primary anastomosis is a firstline treatment for tracheal stenosis. Collaboration is needed between thoracic and ENT surgeons to develop protocols for management of tracheal stenosis.

\section{References}

1. Nair S, Mohan S, Mandal G, Nilakantan A. Tracheal stenosisour experience at a tertiary care centre in India with special regard to cause and management. Indian J Otolaryngol Head Neck Surg. 2014; 66:51-6

2. Stauffer JL, Olson DE, Petty TL. Complications and consequences of endotracheal intubation and tracheostomy: A prospective study of 150 critically ill adult patients. Am J Med. 1981; 70:65-76

3. Grillo HC, Donahue DM, Mathisen DM, Wain JC, Wright CD. Postintubation tracheal stenosis-Treatment and results. J Thorac Cardiovasc Surg. 1995; 109:486-93

4. Bartels S, Mayberry JC, Goldman RK, Askew JA, Wax MK. Tracheal stenosis after percutaneous dilatational tracheostomy. Otolaryngol Head Neck Surg. 2002; 126:58-62

5. Mahanta V, Howson C. Oesophageal ballon tracheoplasty in early tracheal stenosis following percutaneous tracheostomy. Anaesth Intensive Care. 2011; 39:678-81

6. Norwood S, Vallina VL, Short K, Saigusa M, Fernandez MD, McLarty JW. Incidence of tracheal stenosis and other late complications after percutaneous tracheostomy. Ann Surg. 2000; 232:233-41

7. Elsayed H, Mostafa AM, Soliman S, Shoukry T, El-Nori AA, El-Bawab HY. First-line tracheal resection and primary anastomosis for postintubation tracheal stenosis. Ann R Coll Surg Engl. 2016; 98:425-30

8. Sarper A, Ayten A, Eser I, Ozbudak O, Demircan A. Traheal stenosis after tracheostomy or intubation. Texas Heart Institute J. 2005; 32:154-8

9. Kandakure VT, Mishra S, Lahane VJ. Management of post traumatic laryngotracheal stenosis: our experience. Indian J Otolaryngol Head Neck Surg. 2015; 67:255-60

10. Nouraei SM, Nouraei SAR. Assessment of Patients and Outcomes in Laryngotracheal Stenosis. In: Sandhu GS, Nouraei SAR, eds. Laryngeal and Tracheobronchial Stenosis. San Diego: Plural Publishing. 2016; 53-70

11. Dass A, Nagarkar NM, Singhal SK, Verma H. Tracheal t-tube stent for laryngotracheal stenosis: ten year experience. Iranian J Otolaryngol. 2014; 26(74):37-42

12. Mostafa BA, Mbarek CC, Varghese BT. Management. In: Mostafa BE, Mberek CC, Halafawi AE, eds. Tracheal Stenosis: Diagnosis and Treatment. Egypt: Faculty of Medicine Aln Shams University. 2012; 35-52 
13. Negm H, Mosleh M, Fathy H. Circumferential tracheal resection with primary anastomosis for post-intubation tracheal stenosis: study of 24 cases. Eur Arch Otorhinolaryngol. 2013; 270:2709-17
14. Ahn SH, Sung MW, Kim KH. Factors associated with staged reconstruction and successful stoma closure in tracheal resection and end-to-end anastomosis. Arch Otolaryngol Head Neck Surg. 2004; 130:57-62. 